\title{
An unusual injury mechanism of atlantoaxial dislocation: illustrative case
}

\author{
Yakubu Ibrahim, MD, ${ }^{1,2}$ Yiwei Zhao, MD, ${ }^{1,2}$ Wubo Liu, MD, ${ }^{1}$ Suomao Yuan, MD, ${ }^{1,2}$ Yonghao Tian, MD, ${ }^{2}$ Lianlei Wang, MD, PhD, ${ }^{1}$ and \\ Xinyu Liu, MD, PhD ${ }^{1,2}$ \\ ${ }^{1}$ Department of Orthopedics, Qilu Hospital, Shandong University, Jinan, Shandong, China; and ${ }^{2}$ Cheeloo College of Medicine, Shandong University, Jinan, Shandong, China
}

BACKGROUND Atlantoaxial dislocation (AAD) is a rare and potentially life-threatening condition. Various underlying mechanisms of injury are described in the literature. Here, the authors report an unusual nontraumatic injury mechanism of AAD in a 12-year-old patient.

OBSERVATIONS A 12-year-old boy presented with intolerable neck pain and numbness in both upper limbs. The patient's symptoms had started 2 months after the initiation of online classes during the coronavirus disease 2019 pandemic without a history of trauma. He used a computer for personal study and online classes for prolonged hours with no respite. On physical and radiological evaluation, he was diagnosed with AAD. Before surgery, skull traction was applied to reduce the dislocation and posterior C1 lateral mass screw and C2 pedicle screw fixation was performed. An optimal clinical outcome was achieved with no postoperative complications. A preoperative visual analog scale score of 8.0 was reduced to 0 postoperatively.

LESSONS A prolonged fixed neck posture is an unusual underlying cause of AAD. Posterior $\mathrm{C} 1$ lateral mass and $\mathrm{C} 2$ pedicle screw fixation results in an optimal clinical outcome.

\section{https://thejns.org/doi/abs/10.3171/CASE21134}

KEYWORDS atlantoaxial dislocation; atlantoaxial joint; case report; fixed neck posture; atlantoaxial injury mechanism; unusual atlantoaxial dislocation presentation

Atlantoaxial dislocations (AADs) can be grouped into four basic categories based on the direction and plane of the dislocation: anteroposterior, rotatory, central, and mixed dislocations. The atlantoaxial joints (AAJs) form the most mobile segment of the entire spine and hence are more prone to instabilities. The loss of stability between the atlas and the axis leading to the impairment of normal articulation at the C1-2 joint is referred to as an atlantoaxial dislocation.

The various degrees of complex movements that occur at the AAJ include angular motion (flexion and extension), rotation (right and left), and linear motion and translation (anterior and posterior, right, and left). The flexible articulation interaction of $\mathrm{C}$, the dens, and the transverse ligament is responsible for over $50 \%$ of all cervical spine rotation. ${ }^{1,2}$ However, AAJ stability can be disrupted by trauma, congenital anomalies, and idiopathic and inflammatory processes. ${ }^{2}$ Here, we report an unusual mechanism of injury leading to $A A D$ in a 12 -year-old patient.

\section{Illustrative Case}

A 12-year-old boy reported severe neck pain of 2 months' duration. He had first noticed mild neck pain after remaining in a prolonged fixed position in front of a computer. He had little or no respite between classes, and he had 12 to 16 hours of online classes in a day. His neck pain aggravated gradually over time until it became intolerable. He was admitted to the hospital with intractable neck pain, and he underwent physical and radiological examinations. On physical examination, his neck range of motion (ROM) was limited, with mild tenderness and pain to palpation at the level of the cervical spinous process. Numbness in the occipital protuberance at the base of the skull was observed, suggesting C2 dermatome deficit. The findings of both upper- and lower-limb evaluations were normal. The patient's reflexes were normal (bilateral biceps reflex ++ , triceps brachii reflex ++ , bilateral knee reflex ++ , and bilateral ankle reflex ++ ).

ABBREVIATIONS AAD = atlantoaxial dislocation; $A A J=$ atlantoaxial joint; $A D I=$ atlantodental interval; $C T=$ computed tomography; ROM = range of motion; $V A S=$ visual analog scale.

INCLUDE WHEN CITING Published June 14, 2021; DOI: 10.3171/CASE21134.

SUBMITTED March 1, 2021. ACCEPTED March 28, 2021.

(C) 2021 The authors, CC BY-NC-ND 4.0 (http://creativecommons.org/licenses/by-nc-nd/4.0/). 
On radiographs, there was a forward shift of the atlas, the anterior and posterior atlantodental interval (ADI) was widened, and the space between the posterior arch and the $\mathrm{C} 2$ spinous process was widened (Fig. $1 \mathrm{~A}$ and $\mathrm{C}$ ). Magnetic resonance imaging evaluation indicated a distorted AAJ with a posterior and upward shift of the odontoid process compressing the dural sac with a narrowed spinal canal at the level of the foramen magnum. (Fig. $2 \mathrm{C}$ and $\mathrm{D}$ ). A computed tomography (CT) scan showed widened lateral mass spaces with the atlas rotated to the right (Fig. $2 A$ and $B$ ). $A A D$ was the final diagnosis.

On initial presentation, the patient was placed in cervical traction. A weight $(10 \mathrm{~kg}$ ) was placed according to the weight of the patient. Anteroposterior and lateral cervical radiographs were obtained within 48 hours, which showed that the dislocation was reduced partially (Fig. 1B and D).

Two days after admission, the patient was taken to the operating room for definitive surgical management of his injury. The patient was placed in a prone position with continuous skull traction. Intraoperative fluoroscopy showed reduction of his dislocation. However, high atlantoaxial instability was present.

A posterior midline incision extending from the suboccipital area to the spinous process of $\mathrm{C} 3$ was made. The $\mathrm{C} 1$ dorsal arch and C2-3 facet joints were exposed. The medial wall of $\mathrm{C} 1$ laterally and the medial aspect of the transverse foramen at $\mathrm{C} 1$ and $\mathrm{C} 2$ were identified to serve as medial and lateral limits for screw placement. The ventral cortex of the lateral mass midway between the superior and inferior facets of $\mathrm{C} 1$ was penetrated to place the screws.

The C2 pedicles were exposed, and the trajectory of screws was identified lateral to the superior margin of the C2 lamina. The bilateral screws were placed after a drill was used to create the entry point. After satisfactory screw placement was confirmed by fluoroscopy, the screws
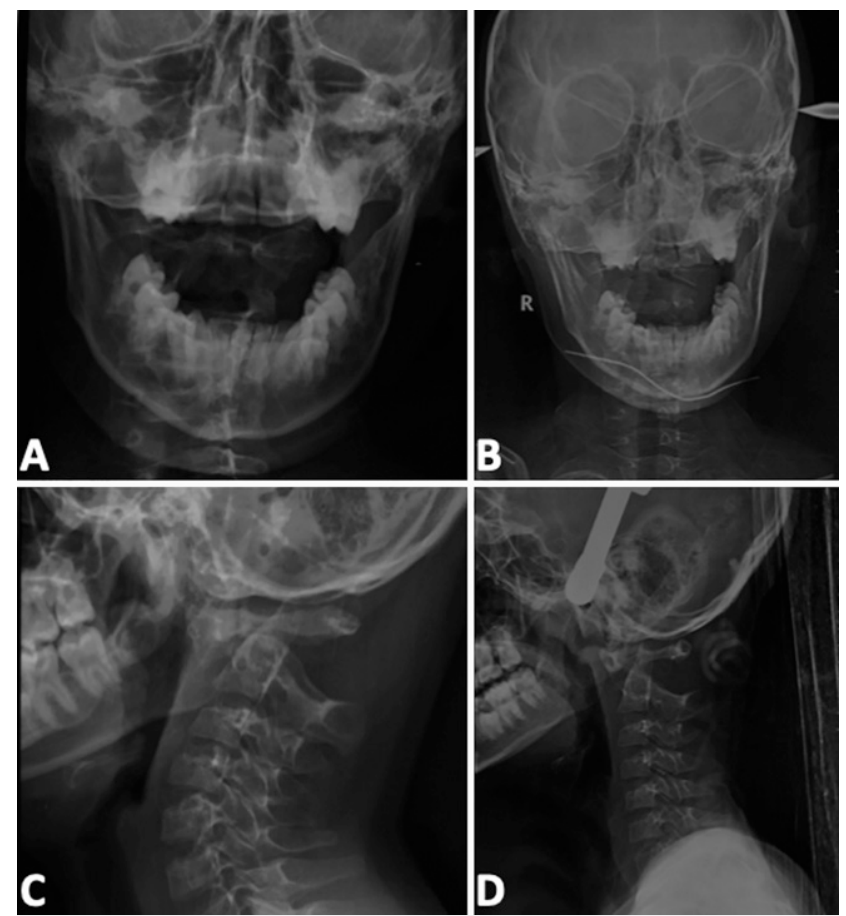

FIG. 1. A-D: Anteroposterior open-mouth and lateral radiographs with and without skull traction showing a forward shift of the atlas, a widened anteroposterior ADI, and the widened space between the posterior arch and $\mathrm{C} 2$ spinous process.

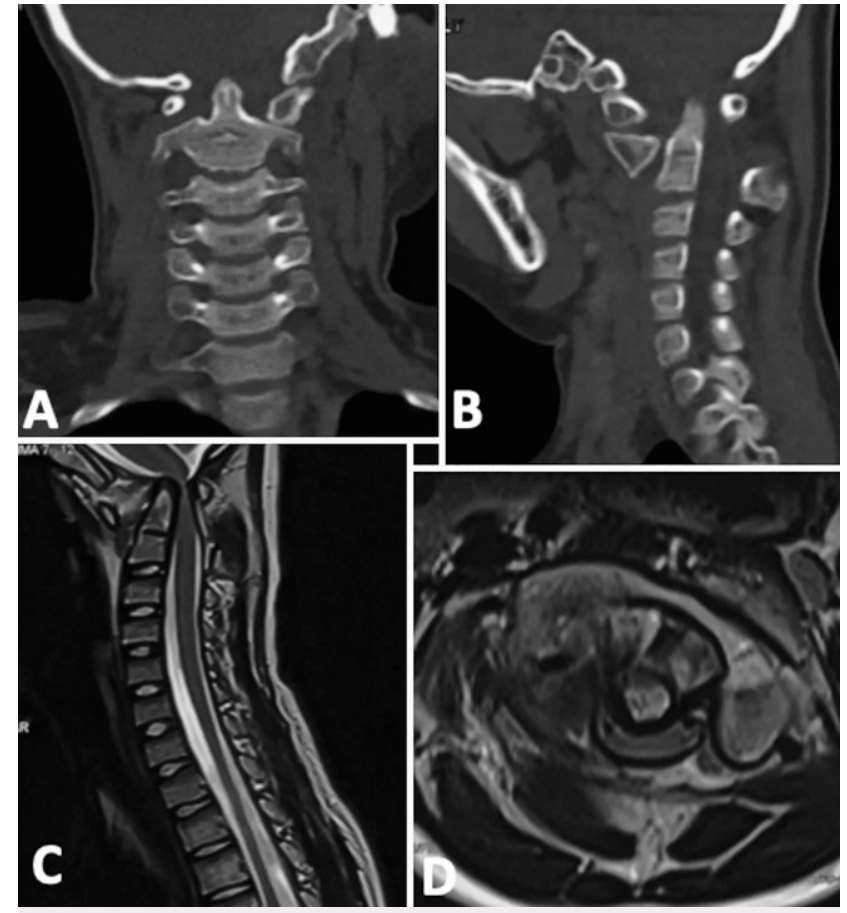

FIG. 2. A: Coronal CT scan showing atlas rotation of the posterior arch to the right. B: Sagittal CT scan showing the right lateral mass rotation to the front of the dens. $C$ and D: Sagittal and axial magnetic resonance images showing distorted AAJ, narrowed spinal canal at the level of the foramen magnum, and spinal cord compression by the posteriorly shifted odontoid process.

were tightened over the rods. An allogenic bone graft was used for arthrodesis. The incision was closed layer by layer. The patient's postoperative course was satisfactory without complications. A postoperative radiograph showed good cervical alignment and
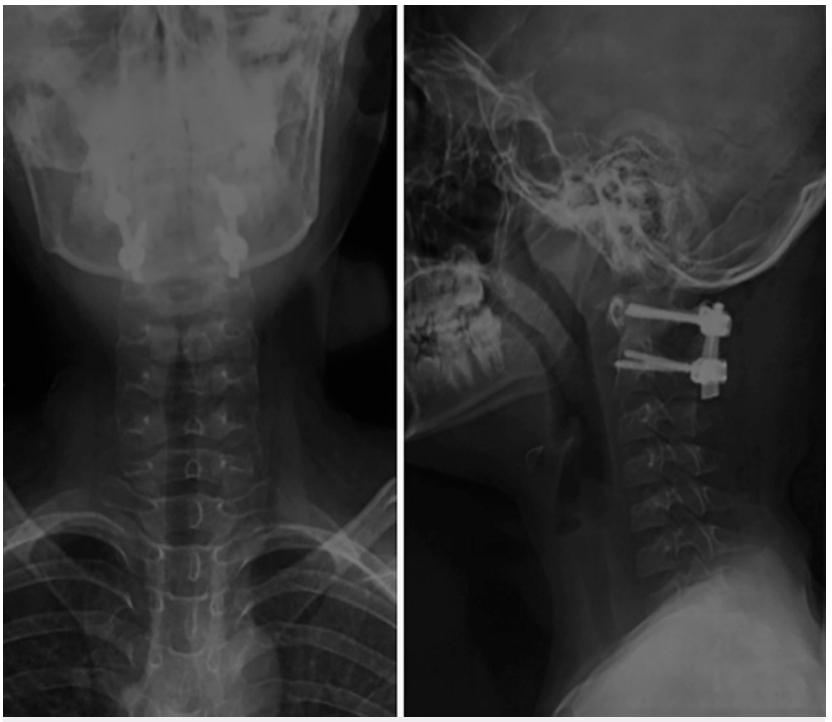

FIG. 3. Postoperative radiographs: anteroposterior (left) and lateral (right) views showing satisfactory cervical alignment with accurate posterior instrumentation. 


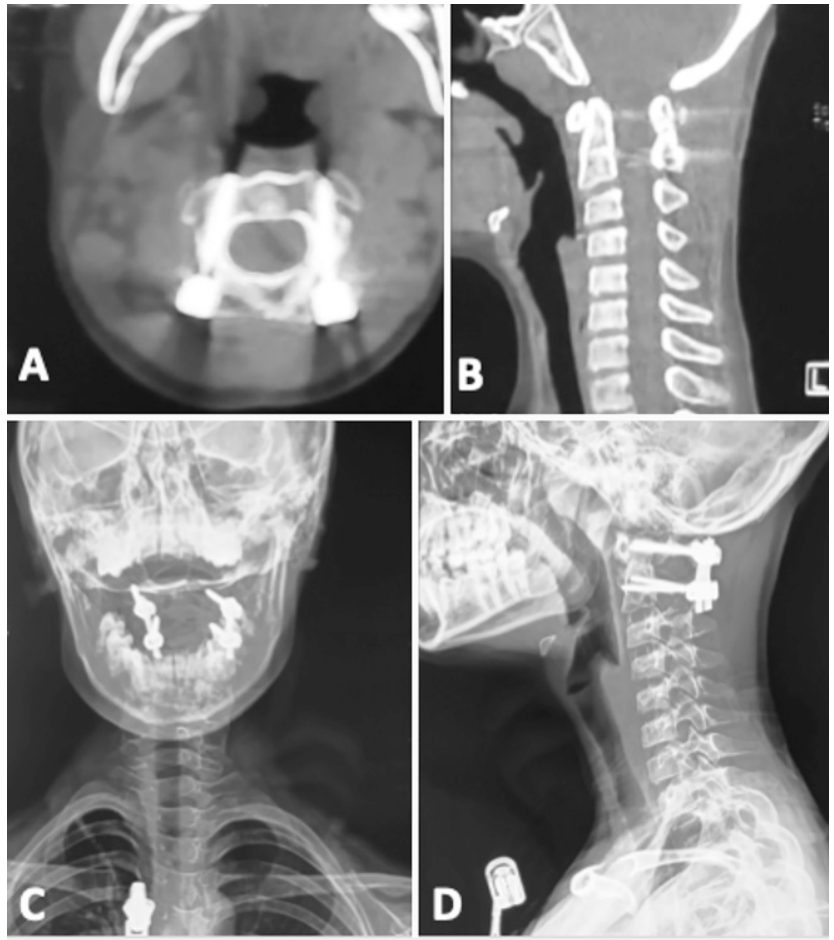

FIG. 4. Radiography at 3-month follow-up. A and B: Axial and sagittal $C T$ scans. $C$ and $D$ : Open-mouth and lateral radiographs showing normal AAJ, ADI, bony alignment, and a satisfactory posterior bone fusion.

correct screw placement (Fig. 3). The patient's visual analog scale (VAS) score significantly improved from 8.0 preoperatively to 0 on postoperative day 2.

Radiological evaluation at 3-month follow-up indicated satisfactory screw positioning and normal cervical alignment (Fig. 4). The patient's VAS score for neck pain was 0.

\section{Discussion}

The etiologies of $A A D$ can vary widely. However, they are generally categorized as traumatic, congenital, and inflammatory processes. ${ }^{3}$ Even though injuries and dysfunction of the AAJ commonly occur, traumatic $A A D$ is rare in the literature. ${ }^{4} A$ traumatic cause of $A A D$ is associated with transverse ligament injuries more often than with osseous injuries. ${ }^{5,6}$

Certain congenital anomalies and chromosomal inherited disorders such as Down syndrome have also been reported to be prone to joint instability due to associated osseous and ligamentous abnormalities. ${ }^{7}$ The laxity of the ligamentous structures leading to the joint instability may be due to a deformed intrinsic collagenous factor forming these supporting ligaments. ${ }^{8}$ Other precipitating causes of $A A D$ include skeletal dysplasias such as Goldenhar syndrome and osseous abnormalities. ${ }^{9-16}$

The patient described in this case had none of the aforementioned predisposing factors, suggesting that his constant fixed posture was the sole precipitating cause of his injury. Although the patient's history and examinations did not reveal ligamentous laxity, the pathologic mechanism can be attributed to atlantoaxial complex ligamentous incompetence due to the patient's constant fixed neck posture during his protracted online classes.
The diagnosis of AAD can be challenging, and there is a lack of consensus regarding both evaluation and treatment. However, once a detailed evaluation is obtained and the diagnosis of $A A D$ is confirmed, the treatment is aimed at correcting cervical spine sagittal alignment and stability. ${ }^{17}$

Both operative and nonoperative modalities can achieve an optimal clinical outcome. However, there is no universally accepted treatment option. ${ }^{14}$

Traction and a cervical collar are often used for children with spontaneous AAD. ${ }^{18,19}$ The present case, however, had symptomatic AAJ instability, and skull traction failed to maintain AAJ stability. Therefore, to achieve C1-2 stability and prevent further neurological deterioration, fixation with fusion was indicated instead of nonoperative treatment. Satisfactory C1-2 stability was achieved through posterior C1-2 fixation and fusion (Fig. 3).

Although a posterior occipitocervical/C1-2 fusion approach is often applied, the rotatory function of the AAJ is significantly affected. ${ }^{20} \mathrm{How}_{\text {- }}$ ever, the benefits of the cervical spine correction and alignment outweigh the limited cervical ROM. Postoperative complications include kyphosis, subaxial subluxation, vertebral artery injury, and degenerative disc disease. $^{21,22}$

\section{Observations}

Although various instigating factors and injury mechanisms of $A A D$ have been described in the literature, trauma remains the major cause of AAD, especially in young patients. ${ }^{3,23,24}$ The C1-2 entity has a complex anatomy, and subtle insults such as fixed neck posture (as observed in this case) can initiate a cascade of neuroprotective responses, disrupting the ligamentous supporting structures and resulting in AAD.

\section{Lessons}

$A A D$ is generally a rare condition but can be life threatening. Here, we have reported the first case of $A A D$ in a 12-year-old boy with an unusual injury presentation. Considering the significant limitations associated with a single case report, any conclusions drawn from this case should remain tentative.

\section{Acknowledgments}

We are grateful to Ryan K. Harrison for proofreading the final manuscript draft.

\section{References}

1. Pang D, Li V. Atlantoaxial rotatory fixation, I: biomechanics of normal rotation at the atlantoaxial joint in children. Neurosurgery. 2004;55(3):614-626.

2. Subin B, Liu JF, Marshall GJ, et al. Transoral anterior decompression and fusion of chronic irreducible atlantoaxial dislocation with spinal cord compression. Spine (Phila Pa 1976). 1995;20(11): 1233-1240.

3. Klimo P Jr, Rao G, Brockmeyer D. Congenital anomalies of the cervical spine. Neurosurg Clin N Am. 2007;18(3):463-478.

4. Venkatesan M, Bhatt R, Newey ML. Traumatic atlantoaxial rotatory subluxation (TAARS) in adults: a report of two cases and literature review. Injury. 2012;43(7):1212-1215.

5. Grauer JN, Shafi B, Hilibrand AS, et al. Proposal of a modified, treatment-oriented classification of odontoid fractures. Spine $\mathrm{J}$. 2005;5(2):123-129.

6. Guo X, Ni B, Zhao W, et al. Biomechanical assessment of bilateral C1 laminar hook and C1-2 transarticular screws and bone graft for atlantoaxial instability. J Spinal Disord Tech. 2009;22(8):578-585. 
7. Curtis BH, Blank S, Fisher RL. Atlantoxial dislocation in Down's syndrome. Report of two patients requiring surgical correction. JAMA. 1968;205(6):464-465.

8. Pueschel SM, Scola FH. Atlantoaxial instability in individuals with Down syndrome: epidemiologic, radiographic, and clinical studies. Pediatrics. 1987;80(4):555-560.

9. Song D, Maher CO. Spinal disorders associated with skeletal dysplasias and syndromes. Neurosurg Clin N Am. 2007;18(3): 499-514.

10. Healey D, Letts $M$, Jarvis JG. Cervical spine instability in children with Goldenhar's syndrome. Can J Surg. 2002;45(5):341-344.

11. Miyoshi K, Nakamura K, Haga N, Mikami Y. Surgical treatment for atlantoaxial subluxation with myelopathy in spondyloepiphyseal dysplasia congenita. Spine (Phila Pa 1976). 2004;29(21): E488-E491.

12. Takeda E, Hashimoto T, Tayama M, et al. Diagnosis of atlantoaxial subluxation in Morquio's syndrome and spondyloepiphyseal dysplasia congenita. Acta Paediatr Jpn. 1991;33(5):633-638.

13. Rizzolo SJ, Cotler JM. Unstable cervical spine injuries: specific treatment approaches. J Am Acad Orthop Surg. 1993;1(1):57-66

14. Wang S, Wang $C$, Yan $M$, et al. Novel surgical classification and treatment strategy for atlantoaxial dislocations. Spine (Phila $\mathrm{Pa}$ 1976). 2013;38(21):E1348-E1356.

15. Salunke P, Behari S, Kirankumar MV, et al. Pediatric congenital atlantoaxial dislocation: differences between the irreducible and reducible varieties. J Neurosurg. 2006;104(2 suppl):115-122.

16. Salunke P, Sharma M, Sodhi HB, et al. Congenital atlantoaxial dislocation: a dynamic process and role of facets in irreducibility. J Neurosurg Spine. 2011;15(6):678-685.

17. Fielding JW, Hawkins RJRS, Ratzan SA. Spine fusion for atlantoaxial instability. J Bone Joint Surg Am. 1976;58(3):400-407.

18. Weisskopf M, Naeve D, Ruf M, et al. Therapeutic options and results following fixed atlantoaxial rotatory dislocations. Eur Spine J. 2005;14(1):61-68.
19. Goel A, Shah A. Atlantoaxial facet locking: treatment by facet manipulation and fixation. Experience in 14 cases. $J$ Neurosurg Spine. 2011;14(1):3-9.

20. Yeom JS, Buchowski JM, Kim HJ, et al. Risk of vertebral artery injury: comparison between C1-C2 transarticular and C2 pedicle screws. Spine J. 2013;13(7):775-785.

21. Matsunaga S, Onishi T, Sakou T. Significance of occipitoaxial angle in subaxial lesion after occipitocervical fusion. Spine (Phila $\mathrm{Pa}$ 1976). 2001;26(2):161-165.

22. Yoshimoto $H$, Ito $M$, Abumi $K$, et al. A retrospective radiographic analysis of subaxial sagittal alignment after posterior C1-C2 fusion. Spine (Phila Pa 1976). 2004;29(2):175-181.

23. Boijsen $\mathrm{E}$. The cervical spinal canal in intraspinal expansive processes. Acta Radiol. 1954;42(2):101-115.

24. Heiden JS, Weiss MH, Rosenberg AW, et al. Management of cervical spinal cord trauma in Southern California. J Neurosurg. 1975;43(6):732-736.

\section{Disclosures}

The authors report no conflict of interest concerning the materials or methods used in this study or the findings specified in this paper.

\section{Author Contributions}

Conception and design: Ibrahim, Yuan, Wang. Acquisition of data: X Liu, Ibrahim, W Liu, Yuan, Tian. Analysis and interpretation of data: Ibrahim, Wang. Drafting the article: Ibrahim. Critically revising the article: Ibrahim, Zhao, W Liu, Yuan. Reviewed submitted version of manuscript: X Liu, Ibrahim, Zhao, W Liu, Yuan, Wang. Approved the final version of the manuscript on behalf of all authors: $X$ Liu. Statistical analysis: Wang. Study supervision: X Liu, Yuan, Wang.

\section{Correspondence}

Xinyu Liu: Qilu Hospital, Shandong University, Shandong, China. newyuliu@163.com. 